\title{
Die vernachlässigte strategische Dimension
}

\author{
Moderne Managementkonzepte gehen davon aus, dass Führungsentscheide in \\ Unternehmen unterschiedliche Ebenen der Komplexität und Reichweite gleich- \\ zeitig berücksichtigen müssen. Ziele und Maßnahmen sind daher nicht nur auf \\ operativer, sondern auch auf strategischer Ebene zu verorten, wenn sie Erfolg \\ haben sollen. Dies gilt auch für Umweltmanagementsysteme. Eine Typologie ihrer \\ möglichen strategischen Dimensionen kann hier weiterhelfen. Sie wird an \\ empirischen Beispielen illustriert.
}

$\mathrm{D}$ Sankt Galler Managementkonzept unerscheidet zwischen einer normativen, einer strategischen und einer operativen Managementebene. Geht es auf normativer Ebene darum, die generellen Ziele, Prinzipien und Regeln für die grundsätzliche Ausrichtung der Unternehmenstätigkeiten festzulegen, so geht es auf strategischer Ebene um den Aufbau und die Pflege von Erfolgspotenzialen und auf operativer Ebene schließlich um die Nutzung solcher - in der Vergangenheit aufgebauter - Potenziale für die Erfolgsrealisierung. Wichtig ist die Erkenntnis, dass ein wirkungsvolles Managementsystem über eine gleichgewichtige Ausprägung von Elementen auf allen drei Ebenen verfiugen muss. Es bedarf insbesondere der Ziele und Maßnahmen nicht nur auf operativer, sondern auch auf strategischer Ebene, da strategische Versäumnisse auch durch noch so große Anstrengungen auf operativer Ebene nicht wieder wettgemacht werden können. Mit anderen Worten: Führt der Weg in die falsche Richtung oder bleibt das Ziel im Dunkeln, dann hilft es nichts, das Tempo zu erhöhen.

Mit dem Aufbau und Einsatz von Umweltmanagementsystemen (UMS) sollten daher immer strategisch relevante Fragestellungen gezielt verknüpft werden. Die strategische Grundfrage lautet: Wozu soll das UMS dienen? Diese Grundfrage lässt sich in Form zweier Teilfragen weiter konkretisieren:

1. Welche Unternehmensziele und -strategien sollen durch das UMS ermöglicht bzw. unterstützt werden?

2. Inwiefern dient das UMS und seine Weiterentwicklung als Instrument der Unternehmensentwicklung?
Im Hinblick auf die Beantwortung dieser Fragen sind die folgenden Aspekte näher zu betrachten:

- Stehen interne oder externe Ziele im Vordergrund?

Welche Anspruchsgruppen werden anvisiert?

Welche Aktivitäten und Maßnahmen sind zu entwickeln? Wann sind sie einzuleiten und bis wann sollen sie greifen?

- Welche Stellen und Bereiche sind in Aufbau und Betrieb des UMS einzubinden?

Welche Erwartungen an die Weiterentwicklung des UMS gibt es?

Ein Blick in die Praxis zeigt, dass UMS oftmals aufgebaut werden, ohne dass man sich explizit über die damit verfolgten strategischen Ziele Rechenschaft ablegt. Man verspürt ein mehr oder weniger offensichtliches strategisches Defizit. Die Folge sind unklare Prioritäten und Enttäuschungen angesichts suboptimaler Resultate.

\section{Strategietypen von UMS}

Um die genannten Fragen zu beantworten, unterscheiden wir in Abbildung 1 zwischen fünf verschiedenen UMS-Typen aber nur zwischen vier strategischen UMS-Ausrichtungen. Sie werden in eine Matrix mit den Dimensionen ,Ansatzpunkt für Maßnahmen“ und „Strategiebezug“ eingeordnet (1).

Der UMS-Typ Infrastruktur ist in der Mitte der Grafik angeordnet, weil er sowohl auf gesellschaftliche wie auf marktliche Anforderungen bezogen sein und sowohl interne wie auch externe Ansatzpunkte aufweisen kann. Bei ihm geht es vor allem darum, im Unternehmen systematisch vorzugehen, somit Transparenz und Ordnung in Abläufe und Systeme zu bekommen. Das UMS ist hier ein Teil der Management-Infrastruktur. Ziele, die hier typischerweise verfolgt werden, sind Systematisierung umweltrelevanter Prozesse und Stärkung der Motivation von Mitarbeitenden. Dieser allgemeinste, grundlegende UMS-Typ verwirklicht im Prinzip nur die Systemanforderungen der ISO-Norm 14001, ohne dass bereits eine inhaltliche strategische Ausrichtung erkennbar wäre. Er lässt sich aber mit unterschiedlichen strategischen Ausrichtungen verknüpfen. Demgegenüber weisen die übrigen UMS-Typen eine klare strategische Ausrichtung auf.

Der UMS-Typ Auditierung weist eine interne Ausrichtung auf und ist auf Ansprüche der Gesellschaft bezogen. Mit Hilfe des UMS sollen hier primär Ziele wie Rechtssicherheit und Risikovorsorge verfolgt werden. Es geht darum, als Unternehmen ökologisch clean zu sein, somit ökologische Schwachpunkte und Risiken frühzeitig zu erkennen und zu beseitigen. Bestehende Märkte und Geschäftstätigkeiten sollen gegenüber ökologischen Ansprüchen und Einschränkungen abgesichert werden. Im Vordergrund stehen dabei Aufga-

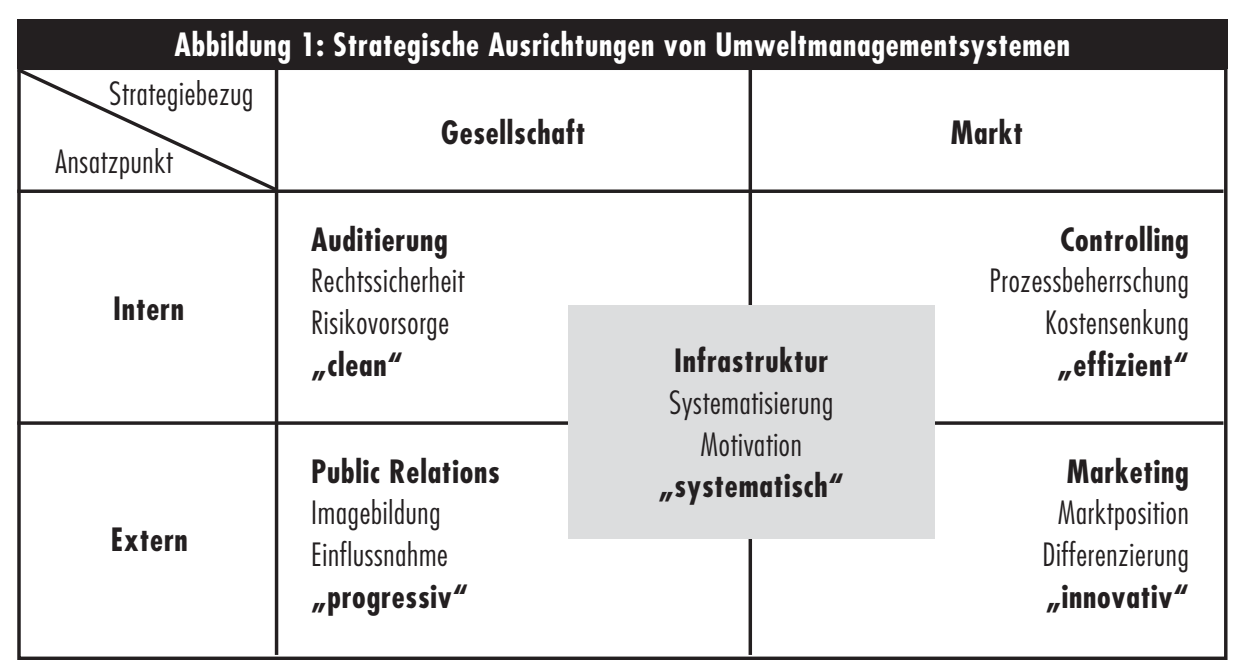

Quelle: eigene Darstellung 
ben der Absicherung, Überwachung und Kontrolle. Dieser Typ findet sich zumeist in risikobehafteten Tätigkeitsfeldern und Branchen wie Chemie, Mineralöl, Energieversorgung oder Transporte.

Der UMS-Typ Controlling weist ebenfalls eine interne Ausrichtung auf, steht aber im Kontext wirtschaftlicher Anforderungen des Marktes. Ökologische Anforderungen oder Rahmenbedingungen sollen hier mit Hilfe eines UMS möglichst effizient, das heißt kostengünstig erfüllt werden. Die typischen Ziele sind hier einerseits die Prozessbeherrschung, andererseits das Aufspüren ökologischer Kostensenkungspotenziale. Solche finden sich vor allem im Bereich des Ressourcen- und Abfallmanagements, den klassischen Ansatzpunkten für Verbesserungen der Öko-Effizienz. Der Einsatzbereich dieses UMS-Typs ist nicht branchenspezifisch bestimmt und sehr breit. Er hängt jedoch von unternehmenspolitischen Entscheidungen ab. Der UMS-Typ Marketing ist auf marktliche Einflüsse bezogen und extern ausgerichtet. Das strategische Ziel besteht hier darin, durch geeignete Maßnahmen bestehende Marktpositionen zu sichern und neue Differenzierungspotenziale zu erschließen. Wird das UMS als Marketinginstrument eingesetzt, dann rücken Maßnahmen bezüglich ökologischer Produkt- und Leistungsinnovationen, aber auch Kommunikations- und Werbemaßnahmen in den Vordergrund. Das UMS dient dann vor allem dazu, ökologisch innovativ zu sein. Der Einsatz dieses UMS-Typs hängt von unternehmensstrategischen Entscheidungen ab. Ein günstiges Umfeld findet er in Bereichen wie Lebensmittel, Textilien, Verkehr, Energieerzeugung, Bauen und Wohnen.

Der letzte UMS-Typ Public Relations (PR) ist auf die Gesellschaft bezogen und weist externe Ansatzpunkte für Maßnahmen auf. Typische UMS-Ziele sind die Image- und Vertrauensbildung in der Öffentlichkeit oder eine gezielte Einflussnahme auf die Meinungsbildung in Politik und Öffentlichkeit. Das UMS dient hier dazu, progressiv zu sein oder zumindest zu wirken. Es geht zum einen um eine ökologische Selbstdarstellung und Profilierung, zum anderen um eine Legitimierung als anerkannter Akteur in öffentlichen und politischen Entscheidungsprozessen. Dieser UMS-Typ findet sich häufig bei öffentlich stark exponierten Unternehmen, die entweder durch ihre Größe, wie Großbanken, oder aber durch ihre Tätigkeit in besonderem Maße auf Vertrauen angewiesen sind. Beispiele hierfür sind Fluggesellschaften und Gentechnik-Anwender.

Diese Unterscheidung nach UMS-Typen und Ausrichtungen hat idealtypischen Charakter. Sie zeigt auf, welche „reinen“ Formen sich unterscheiden lassen und macht bewusst, zwischen welchen Möglichkeiten bei UMS-Aufbau und -Ausrichtung gewählt werden kann. In der Realität finden sich zumeist kombinierte oder gemischte Formen. UMS werden dabei für mehrere Zwecke gleichzeitig - oder hintereinander - eingesetzt. Grenzen der Kombination ergeben sich daraus, dass unterschiedliche Ausrichtungen auch andere Schwergewichte bei Ausgestaltung und Betrieb des UMS bedingen.

\section{Empirische Illustrationen}

Anhand ausgewählter empirischer Ergebnisse einer schriftlichen Befragung aller ISO 14001-zertifizierten Unternehmen in der Schweiz soll die strategische Ausrichtung von UMS näher beleuchtet werden (2). Die Umweltmanager wurden einerseits nach den Entscheidungsgründen für die UMS-Einführung gefragt, andererseits nach dem tatsächlich eingetretenen Nutzen. Die Ergebnisse zeigen, dass UMS vor allem aus Gründen der Imagebildung und wegen des angestrebten Zertifikats eingeführt werden. Man verspricht sich somit für seine Leistungen vor allem Anerkennung von Außen. Die strategische Ausrichtung entspricht dem UMS-Typ Public Relations. Interne Wirkungen wie die Systematisierung bestehender Maßnahmen (UMS-Typ Infrastruktur) und Risikovorsorge/ Haftungsvermeidung (UMS-Typ Auditierung) sowie Wirkungen auf dem Markt in Form einer Verbesserung der Marktposition oder Stärkung der Innovationsfähigkeit (UMS-Typ Marketing) folgen als weitere wichtige Gründe auf Plätzen 3 bis 6. Das Erkennen von Kostensenkungspotenzialen (UMS-Typ Controlling) findet sich überraschenderweise erst auf Platz 7 vor den erwarteten Erleichterungen im Umgang mit Behörden

Stellt man diesem erwarteten Nutzen den ebenfalls erhobenen eingetretenen Nutzen gegenüber, so ergeben sich auffällige Differenzen. An der Spitze der Rangliste steht hier die Systematisierung bestehender Umweltmaßnahmen. Gut drei Viertel der Befragten sehen hierin einen großen Nutzen für die Unternehmung. Auf den Plätzen 2 und 3 folgen die Sicherung der Rechtskonformität (59 Prozent) und die Risikovorsorge (58 Prozent), wiederum deutlich vor den Kostensenkungspotenzialen. Im Vergleich mit diesen internen Nutzenkategorien wird der externe Nutzen geringer eingeschätzt; oft mit Werten von unter 50 Prozent bezüglich Unternehmensimage in der Öffentlichkeit, Verhältnis zu den Vollzugsbehörden, Stärkung der Innovationsfähigkeit oder Verbesserung der Marktposition.

\section{- Diskrepanz zu den Erwartungen}

Die hier zum Ausdruck kommenden bedeutenden Unterschiede zwischen dem erwarteten und dem eingetretenen Nutzen verweisen darauf, dass entweder die Erwartungen unrealistisch sind oder die Umsetzung mangelhaft. Obwohl beides eine Rolle spielen dürfte, belegen unsere Ergebnisse insbesondere die letztere These.

Mit Blick auf die primär angestrebten, aber nur zum Teil erreichten Imagevorteile lässt sich feststellen: Man verspricht sich Anerkennung von Außen und findet Systematik und Sicherheit im Innern! UMS erweisen sich offenbar im Hinblick auf die Systematisierung und Kontrolle umweltrelevanter Prozesse als enttäuschungssicher, während das Erreichen von Innovationen und Markterfolgen als große, bisher aber unerfuillte Herausforderung für den UMS-Einsatz anzusehen ist. Die Gründe hierfür sollten jedoch weniger in der Struktur von UMS oder deren Normen gesucht werden. Sie liegen eher in der inkonsistenten Umsetzung der angestrebten Strategien, die bislang in stark ausgeprägtem Maße auf interne Prozesse und Strukturen ausgerichtet ist.

\section{Anmerkungen}

(1) Diese Typologie ist angelehnt an die ähnliche Typologie ökologischer Wettbewerbsstrategien in Dyllick, T./ Belz, F./ Schneidewind, U.: Ökologie und Wettbewerbsfähigkeit, München/ Zürich 1997.

(2) Im Rahmen eines Forschungsprojekts am IWÖ-HSG der Universität St. Gallen wurden im Frühjahr 1999 alle ISO 14001-zertifizierten Unternehmen in der Schweiz zum Stand der UMS-Praxis befragt. Die Auswertung basiert auf 158 ausgewerteten Fragebögen, was einem Rücklauf von 54 Prozent entspricht. Die kompletten Ergebnisse und Vorschläge zur Weiterentwicklung von UMS finden sich in: Dyllick, T./ Hamschmidt, J.: Wirksamkeit und Leistung von Umweltmanagementsystemen - Eine Untersuchung von ISO 14001 zertifizierten Unternehmen in der Schweiz, VdF-Verlag, Zürich 2000. Aktuelle Informationen - u.a. auch zum jeweiligen Stand der ISO 14001-Zertifizierungen in der Schweiz - finden sich unter: www.iwoe.unisg.ch 
(c) 20I0 Authors; licensee IÖW and oekom verlag. This is an article distributed under the terms of the Creative Commons Attribution Non-Commercial No Derivates License (http://creativecommons.org/licenses/by-nc-nd/3.o/), which permits unrestricted use, distribution, and reproduction in any medium, provided the original work is properly cited. 